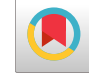

\title{
Lipoglycopeptide Antibiotics: Reliable Fighters Against Gram-Positive Pathogens
}

\author{
Haleh Bagheri ${ }^{1}$, Bahram Sohrabi ${ }^{2}$, Sara Salarian ${ }^{3}$ and Bahador Bagheri ${ }^{4,}{ }^{*}$ \\ ${ }^{1}$ Department of Pharmacology, Faculty of Medicine, CHU Toulouse, 31000-France \\ ${ }^{2}$ Department of Cardiovascular Diseases, Tabriz University of Medical Sciences, Tabriz, Iran \\ ${ }^{3}$ Department of Intensive Care, Shahid Beheshti University of Medical Sciences, Tehran, Iran \\ ${ }^{4}$ Cancer Research Center and Department of Pharmacology, Semnan University of Medical Sciences, Semnan, Iran \\ "Corresponding author: Department of Pharmacology, School of Medicine, Semnan University of Medical Sciences, Semnan, Iran. Email: bahadordvm@yahoo.com
}

Received 2017 August 06; Revised 2018 July 19; Accepted 2018 August 12.

\begin{abstract}
Multi-drug resistant (MDR) strains of staphylococci are usually difficult to treat. Vancomycin has had a time-honored niche in treating MDR Staphylococcus strains; however, during recent years, many clinical failures have been reported worldwide. Since 2014, new semisynthetic lipoglycopeptides antibiotics have been introduced to combat methicillin-resistant Staphylococcus aureus (MRSA), vancomycin-intermediate Staphylococcus aureus (VISA), vancomycin-resistant Staphylococcus aureus (VRSA), and vancomycin-resistant enterococci (VRE). They include dalbavancin, oritavancin, and telavancin. These semisynthetic lipoglycopeptides have a considerable efficacy against MDR Staphylococcus strains. Due to the presence of a lipid side chain, the half-life is prolonged and enables them to anchor the cell membrane of a pathogen. Lipoglycopeptides display a greater potency and more consistent activity against all species of staphylococci than vancomycin. Among them, oritavancin is active against MRSA, VISA, and VRSA. However, dalbavancin and telavancin have activities against MRSA and VISA. Dalbavancin is used once weekly, telavancin is used daily, and oritavancin is usually administered one dose per treatment. Compared to vancomycin, these semisynthetic lipoglycopeptides have longer half-lives with a lower minimum inhibitory concentration (MIC) and fast bactericidal activities. In addition, lipoglycopeptides have concentration-dependent effects in vivo and in vitro. In the present paper, we review the structure, mechanism of action, microbiology, indications, safety, and important interactions of dalbavancin, oritavancin, and telavancin.
\end{abstract}

Keywords: Skin Infections, Antibiotic, Lipoglycopeptide, Resistance

\section{Literature Search}

A literature search was done on Medline and EMBASE using relevant keywords like lipoglycopeptides, vancomycin, telavancin, dalbavancin, oritavancin, MRSA, VRSA, pneumonia, and skin infection in keywords, titles, or abstracts. Additional references were found from bibliographies of the selected papers. Relevant medical texts were checked when required. Randomized controlled trials and other types of studies were considered.

\subsection{Structure}

Glycopeptides have a common heptapeptide core, which enables them to inhibit the cell wall synthesis (1). The peptide backbone of glycopeptides forms the D-alanylD-alanine binding site that is important for their antimicrobial activities (2). Glycopeptides can bind to C-terminal D-alanyl-D-alanine (D-Ala-D-Ala) of cell wall precursor units and disrupt the polymerization of $\mathrm{N}$-acetylglucosamine and $\mathrm{N}$-acetylmuramic acid. Because of their large molecular size, they are unable to penetrate the outer membrane of Gram-negative bacteria (3). All three lipoglycopeptides contain lipophilic side chains, which prolong their halflives and increase their activities against Gram-positive cocci (4). The length of the lipophilic side chain is important; an increase in the chain length increases the activity of the agent against enterococci but it reduces the activity against MRSA (5, 6). Dalbavancin is a semisynthetic derivative of teicoplanin. Modifications include the removal or substitution of sugars and derivatization of the functional groups such as the carboxy group of amino acid 7 (the Cterminus of the peptide), the N-terminus of the peptide, and different hydroxy groups. All modifications are at sites that do not directly affect the D-alanyl-D-alanine binding pocket (7). Oritavancin is a synthetic derivative of naturally occurring glycopeptides chloroeremomycin. N-alkylp-chlorophenyl benzyl substituent improves the activity of oritavancin against both vancomycin-susceptible entero-

Copyright (c) 2018, Archives of Clinical Infectious Diseases. This is an open-access article distributed under the terms of the Creative Commons Attribution-NonCommercial 4.0 International License (http://creativecommons.org/licenses/by-nc/4.0/) which permits copy and redistribute the material just in noncommercial usages, provided the original work is properly cited. 
cocci (VSE) and VRE (8). Telavancin is a derivative of vancomycin. Unlike vancomycin, it has hydrophobic and hydrophilic moieties enabling it to be active against VRE and MRSA (9).

\subsection{Mechanism of Action}

In general, lipoglycopeptides are the inhibitors of the cell wall (10). Cell wall synthesis is impeded in a different way compared to beta-lactams. Lipoglycopeptides are linked with D-alanyl-D-alanine terminus of the peptidoglycan precursor binding to the $\mathrm{C} 55$-lipid transporter (bactoprenol). The drug-amino acid complex effectively inhibits transglycosylase and thus the polymerization of $\mathrm{N}$-acetyl muramic acid and $\mathrm{N}$-acetyl glucosamine is impeded. The linear polymers must be cross-linked by transpeptidase to form a strong cell wall. In addition to the inhibition of transglycosylation, lipoglycopeptides are able to inhibit transpeptidation (11-13). Compared to vancomycin, oritavancin and telavancin exhibit a dual mechanism of action by the inhibition of transpeptidation. It has been shown that telavancin and oritavancin are more potent inhibitors of transpeptidase than vancomycin. Moreover, telavancin and oritavancin are able to disrupt the integrity of bacterial membrane and increase the permeability by the hydrophobic tail. Membrane destabilization by dalbavancin has not been shown yet (11,14-16). Of note, unlike telavancin and dalbavancin, oritavancin has a unique property to inhibit D-alanyl-D -lactate in VRE and VRSA, which is of great importance in clinical practice. D -alanyl-D -lactate can reduce the binding affinity of vancomycin by 1000 times (1719).

\subsection{Microbiology}

As stated, lipoglycopeptides are active against Grampositive bacteria with varying efficacy (20). In general, they have a lower MIC than vancomycin against MRSA, VISA, VRSA, VISA, Staphylococcus epidermidis, vancomycinsusceptible enterococci (VSE), VRE, Streptococcus spp., and Clostriudium spp. (21). From a microbiological point of view, they have important differences; dalbavancin has an excellent activity against MRSA and VISA with a poor activity against VRE. Its effects against Clostridium spp. is comparable with that of vancomycin (22). Oritavancin has the widest spectrum against Gram-positives. Oritavancin is 16to 32-fold more potent than vancomycin against MRSA. It displays a potent activity against VISA and VRE. Oritavancin has the lowest MIC against S. pneumonia and penicillinresistant phenotypes (23). Like dalbavancin, telavancin displays a similar activity for MRSA and VISA with the lowest MIC against Clostridium spp. (24).

\subsection{Indications}

Compared to vancomycin, lipoglycopeptides are more potent and exhibit a faster bactericidal activity against important pathogens including MRSA, VRSA, and VRE. In addition to these benefits, they have longer half-lives affecting the frequency of administration, and perhaps the side effects $(25,26)$. Until date, several clinical trials have been performed to evaluate the efficacy and safety of lipoglycopeptides. At present, lipoglycopeptides are considered useful choices for the treatment of skin and skin structure infections. Of note, telavancin has been introduced as an alternative in the therapy of hospital-acquired pneumonia including ventilator-associated bacterial pneumonia caused by susceptible organisms (25-27). Dalbavancin is administered either at a one-dose regimen of $1500 \mathrm{mg}$ IV or at a two-dose regimen of $1000 \mathrm{mg}$ IV followed one week later by $500 \mathrm{mg}$ IV. The infusion time is $30 \mathrm{~min}$ utes (28). Oritavancin is used as a single $1200 \mathrm{mg}$ dose over three-hour IV infusion (29). Telavancin is given 10 $\mathrm{mg} / \mathrm{kg}$ IV every day for 7 - 14 days. Its infusion time is one hour (30). Seltzer et al. performed an open-label, randomized, controlled, Phase II multi-center trial examining one-dose and two-dose dalbavancin versus standard-ofcare therapy for the treatment of skin and soft tissue infection (SSTI) including vancomycin, cephalosporins, clindamycin, piperacillin-tazobactam, or linezolid. 20 patients were given a single $1500 \mathrm{mg}$ intravenous dose of dalbavancin, 21 patients were given a $1000 \mathrm{mg}$ dose of dalbavancin followed by a $500 \mathrm{mg}$ dose 7 days later, and $21 \mathrm{pa}$ tients were administrated the comparator therapy for 7 to 21 days (21 patients). On day 10 and day 20, and on the last day of treatment, the clinical response was examined for one-dose dalbavancin, two-dose dalbavancin and for the comparator regimens, respectively. The success rates were $75 \%$ (15 out of 20 ) for one-dose dalbavancin, $91 \%$ (19 out of 21) for two-dose dalbavancin, and $81 \%$ (17 out of 21 ) for the comparator therapy (31). In addition, Jauregui et al. compared dalbavancin and linezolid efficacy for the treatment of skin and skin structure infection. Dalbavancin group was composed of 571 patients receiving a 1000-mg dose on day one of therapy and a second dose of $500 \mathrm{mg}$ on day 8 . The linezolid group was composed of 283 patients receiving $600 \mathrm{mg}$ twice daily for 14 days, with at least 24 hours of initial intravenous therapy. The clinical response was measured on day 14 ( \pm 2 days). The success rates were $88.9 \%$ and $91.2 \%$, respectively, for dalbavancin and linezolid groups. Two doses of dalbavancin were as effective and as well tolerated as twice daily doses of linezolid (32). A phase II, multicenter, randomized, double-blind, parallel group, active-comparator study named SIMPLIFI was conducted by Dunbar to evaluate the oritavancin efficacy for the treatment of complicated skin and skin structure in- 
fections (cSSSI). Treatment groups were daily (100 patients, $200 \mathrm{mg}$ daily for 3 - 7 days), infrequent (103 patients, 800 $\mathrm{mg}$ on day 1 with an optional $400 \mathrm{mg}$ on day 5), and singledose (99 patients, $1200 \mathrm{mg}$ a one-time dose) groups. The clinical response was measured on days 21 - 29, decided by the investigator. This investigation showed that the single dose of oritavancin was as efficacious as a daily dose and had a similar safety profile (33). Furthermore, the efficacy of a single dose of oritavancin and multi-doses of vancomycin were tested in two pivotal double-blind, randomized phase III studies, SOLO I and II; 1910 subjects with acute bacterial skin and skin structure infections (ABSSI) received a single 1200-mg dose of oritavancin or $1 \mathrm{~g}$ dose of vancomycin for 7 - 10 days. This study confirmed that oritavancin was non-inferior to vancomycin for the primary endpoint at early clinical evaluation $(80.1 \%$ for oritavancin vs. $82.9 \%$ for vancomycin), the clinical cure endpoint at post-therapy evaluation ( $82.7 \%$ vs. $80.5 \%$, respectively), and $a \geq 20 \%$ reduction in lesion size endpoint at early clinical evaluation ( $85.9 \%$ vs. $85.3 \%$, respectively) (34, 35 ). Of note, the efficacy and safety of lipoglycopeptides have not been investigated in children, yet. For this reason, a phase I, multicenter, open-label, pharmacokinetics, safety, and tolerability study of oritavancin in pediatric patients who are younger than 18 years with suspected or confirmed Gram-positive bacterial infections is recruiting. Approximately, 48 patients will be enrolled at 10 - 15 centers in the United States. The starting dose will be IV oritavancin $15 \mathrm{mg} / \mathrm{kg}$. The safety, tolerability, and PK data will be reviewed to determine the appropriate dose (36). Unlike oritavancin and dalbavancin, telavancin can be used as an alternative drug in pneumonia and in cSSSIs. In the ATTAIN study, two methodologically identical, double-blind studies were done in patients with hospital-acquired pneumonia (HAP) due to Gram-positive pathogens (37). 1503 patients received telavancin ( $10 \mathrm{mg} / \mathrm{kg}$ every 24 hours) or vancomycin ( 1 g every 12 hours) for 7 - 21 days. In the pooled clinically evaluable population, cure rates were $82.4 \%$ with telavancin and $80.7 \%$ with vancomycin. The adverse events were comparable between the treatment groups. The mortality rates for telavancin and vancomycin were $18.5 \%$ and $21.5 \%$, respectively. Increases in serum creatinine levels were more common in the telavancin group (16\% vs. $10 \%$ ), which is important in patients with moderate to severe renal impairment. Overall, it was shown in the ATTAIN study that telavancin is non-inferior to vancomycin for treating HAP. In the ATLAS-1 and ATLAS-2 trials, two parallel, randomized, double-blind, active-control, phase 3 studies were performed in 1867 patients with complicated skin and skinstructure infections. Patients were given telavancin (10 $\mathrm{mg} / \mathrm{kg}$ every 24 hours) or vancomycin ( $1 \mathrm{~g}$ every 12 hours). After the receipt of the last antibiotic dose, success was achieved as $88 \%$ in the telavancin group and $87 \%$ in the vancomycin group at 7 - 14 days after the last administration of drugs. Therapy discontinued because of adverse events in $8 \%$ and $6 \%$ of patients who received telavancin and vancomycin, respectively. Except for mild gastrointestinal upset and foamy urine in the telavancin group and pruritus in the vancomycin group, the adverse events were similar. This work demonstrated that telavancin given once daily is at least as effective as vancomycin for the treatment of patients with complicated skin and skin-structure infections, including those infected with $\operatorname{MRSA}(38,39)$. Besides FDA approved indications, some new case reports have provided limited experiences in other situations caused by Gram-positive pathogens. A recent case study reported uselessness of four weeks of treatment with dalbavancin in a woman with infective endocarditis (40). In addition, an acceptable clinical response to oritavancin has been recently reported in a patient with osteomyelitis (41).

\subsection{Safety}

In general, lipoglycopeptides have acceptable safety profiles. The most frequently reported side effect is gastrointestinal upset. Of note, taste disturbance, nausea, and vomiting are more common than diarrhea (35-42). However, dalbavancin administration is associated with a higher rate of nausea and vomiting compared to dalbavancina and oritavancin. Another common side effect of telavancin is foamy urine that is not dangerous. Dizziness, pruritus, and rash are less common side effects seen with telavancin (43). Mild and reversible ototoxicity has been reported in a study (44). Reversible rises in creatinine levels and increases in $\mathrm{QT}_{\mathrm{C}}$ interval have been observed in FAST study (45). For this reason, co-administration of telavancin with drugs that can increase $\mathrm{QT}_{\mathrm{C}}$ interval should be avoided. Amiodarone, procainamide, disopyramide, sotalol, ibutilide, ondansetron, and cisapride are well-documented drugs associated with an increase in $\mathrm{QT}_{\mathrm{C}}$ interval (46). Telavancin should not be prescribed for patients with pre-existing moderate to severe renal impairment unless benefits outweigh the risks. Nausea, headache, and vomiting are the most common side effects seen with oritavancin use. In addition, a slight rise in hepatic transaminases, phlebitis, and tachycardia are rarely reported. Unlike telavancin, it does not need a dose adjustment in renal impairment. It is noteworthy that oritavancin is a weak inducer of CYP3A4 and CYP2D6 and a weak inhibitor of CYP2C9 and CYP2C12. The metabolism of carbamazepine, clonidine, and disopyramide are significantly reduced by concomitant administration of oritavancin. On the other hand, the levels of phenytoin, warfarin, and valproic acid will increase if they are used with oritavancin $(45,47)$. Dalbavancin has a lower rate of gastrointestinal 
problems compared to oritavancin and telavancin. Pyrexia and rash have been rarely seen. Unlike other lipoglycopeptides, dalbavancin is associated with hematotoxicity, in particular, leukopenia. Anemia, thrombocytopenia, and hemorrhage have been noticed very rarely in patients treated with dalbavancin. Reported cases of leukopenia have had spontaneous cure (48). Until date, no clinical interaction has been observed for dalbavancin either major or minor. Of note, the pregnancy category for all three lipoglycopeptides is $\mathrm{C}$, and it is not documented whether they are distributed in milk.

\section{Conclusions}

In conclusion, dalbavancin, oritavancin, and telavancin offer important alternatives in skin infections. Dalbavancin is a promising agent in pneumonia. High quality clinical studies on the efficacy and safety in addition to clinical experience can promote them as preferred antibiotics against Gram-positive pathogens. They are welcome agents in other serious infections as add-on therapy.

\section{Acknowledgments}

The author wishes to thank Professor Garjani for his help.

\section{References}

1. Allen NE, LeTourneau DL, Hobbs JN, Jr. Molecular interactions of a semisynthetic glycopeptide antibiotic with D-alanyl-D-alanine and Dalanyl-D-lactate residues. Antimicrob Agents Chemother. 1997;41(1):6671. [PubMed: 8980756]. [PubMed Central: PMC163661].

2. Allen NE, LeTourneau DL, Hobbs JN, Jr. The role of hydrophobic side chains as determinants of antibacterial activity of semisynthetic glycopeptide antibiotics. J Antibiot (Tokyo). 1997;50(8):677-84. doi: 10.7164/antibiotics.50.677. [PubMed: 9315081].

3. Allen NE, LeTourneau DL, Hobbs JN, Jr, Thompson RC. Hexapeptide derivatives of glycopeptide antibiotics: Tools for mechanism of action studies. Antimicrob Agents Chemother. 2002;46(8):2344-8. doi: 10.1128/AAC.46.8.2344-2348.2002. [PubMed: 12121903]. [PubMed Central: PMC127376].

4. Nicolaou KC, Boddy CN, Brase S, Winssinger N. Chemistry, biology, and medicine of the glycopeptide antibiotics. Angew Chem Int Ed Engl. 1999;38(15):2096-152. doi: 10.1002/(SICI)15213773(19990802)38:15<2096::AID-ANIE2096>3.0.CO;2-F. [PubMed: 10425471].

5. Thaker MN, Wright GD. Opportunities for synthetic biology in antibiotics: Expanding glycopeptide chemical diversity. ACS Synth Biol. 2015;4(3):195-206. doi: 10.1021/sb300092n. [PubMed: 23654249]. [PubMed Central: PMC4384835].

6. Kalan L, Perry J, Koteva K, Thaker M, Wright G. Glycopeptide sulfation evades resistance. J Bacteriol. 2013;195(1):167-71. doi: 10.1128/JB.01617-12. [PubMed: 23104813]. [PubMed Central: PMC3536170].

7. Cooper MA, Williams DH. Binding of glycopeptide antibiotics to a model of a vancomycin-resistant bacterium. Chem Biol. 1999;6(12):891-9. doi: 10.1016/S1074-5521(00)80008-3. [PubMed: 10631517].
8. Van Bambeke F. Glycopeptides and glycodepsipeptides in clinical development: A comparative review of their antibacterial spectrum, pharmacokinetics and clinical efficacy. Curr Opin Investig Drugs. 2006;7(8):740-9. [PubMed:16955686].

9. Jeya M, Moon HJ, Lee KM, Kim IW, Lee JK. Glycopeptide antibiotics and their novel semi-synthetic derivatives. Curr Pharm Biotechnol. 2011;12(8):1194-204. doi: 10.2174/138920111796117382. [PubMed 21470154].

10. Allen NE, Nicas TI. Mechanism of action of oritavancin and related glycopeptide antibiotics. FEMS Microbiol Rev. 2003;26(5):511-32. doi: 10.1111/j.1574-6976.2003.tb00628.x. [PubMed: 12586393].

11. Belley A, Harris R, Beveridge T, Parr TR, Moeck G. Differential targeting of cell wall assembly systems by oritavancin. 47th Interscience Conference on Antimicrobial Agents and Chemotherapy (ICAAC). 17-20 September 2007; Chicago, IL. Washington, DC. ASM Press; 2007.

12. Kim SJ, Cegelski L, Stueber D, Singh M, Dietrich E, Tanaka KS, et al. Oritavancin exhibits dual mode of action to inhibit cell-wall biosynthesis in Staphylococcus aureus. J Mol Biol. 2008;377(1):281-93. doi: 10.1016/j.jmb.2008.01.031. [PubMed: 18258256]. [PubMed Central: PMC2276640].

13. Belley A, McKay GA, Arhin FF, Sarmiento I, Beaulieu S, Fadhil I, et al. Oritavancin disrupts membrane integrity of Staphylococcus aureus and vancomycin-resistant enterococci to effect rapid bacterial killing. Antimicrob Agents Chemother. 2010;54(12):5369-71. doi: 10.1128/AAC.00760-10. [PubMed: 20876372]. [PubMed Central: PMC2981232].

14. Saravolatz LD, Stein GE, Johnson LB. Telavancin: A novel lipoglycopeptide. Clin Infect Dis. 2009;49(12):1908-14. doi: 10.1086/648438. [PubMed: 19911938].

15. Mendes RE, Farrell DJ, Sader HS, Jones RN. Oritavancin microbiologic features and activity results from the surveillance program in the United States. Clin Infect Dis. 2012;54 Suppl 3:S203-13. doi: 10.1093/cid/cir923. [PubMed: 22431850].

16. Biedenbach DJ, Bell JM, Sader HS, Turnidge JD, Jones RN. Activities of dalbavancin against a worldwide collection of 81,673 gram-positive bacterial isolates. Antimicrob Agents Chemother. 2009;53(3):1260-3. doi: 10.1128/AAC.01453-08. [PubMed: 19124664]. [PubMed Central: PMC2650578].

17. Gander S, Kinnaird A, Finch R. Telavancin: In vitro activity against staphylococci in a biofilm model. J Antimicrob Chemother. 2005;56(2):337-43. doi: 10.1093/jac/dki198. [PubMed: 15972312].

18. Van Bambeke F, Van Laethem Y, Courvalin P, Tulkens PM. Glycopeptide antibiotics: From conventional molecules to new derivatives. Drugs. 2004;64(9):913-36. doi: 10.2165/00003495-200464090-00001. [PubMed: 15101783].

19. Guskey MT, Tsuji BT. A comparative review of the lipoglycopeptides: oritavancin, dalbavancin, and telavancin. Pharmacotherapy. 2010;30(1):80-94. doi: 10.1592/phco.30.1.80. [PubMed: 20030476].

20. Arhin FF, Draghi DC, Pillar CM, Parr TR, Jr, Moeck G, Sahm DF. Comparative in vitro activity profile of oritavancin against recent gram-positive clinical isolates. Antimicrob Agents Chemother. 2009;53(11):4762-71. doi: 10.1128/AAC.00952-09. [PubMed: 19738026]. [PubMed Central: PMC2772347].

21. Badal R, Bouchillon S, Hoban D, Hawser S, Johnson A, Hackel M. A multicenterevaluation of in vitro activity of oritavancin and comparators againststaphylococci, enterococci and streptococci-the Orion study. 19th European Congress on Clinical Microbiology and Infectious Diseases. 16-19 May 2009; Helsinki, Finland. Basel, Switzerland. European Society of Clinical Microbiology and Infectious Diseases; 2009.

22. Arhin FF, Kurepina N, Sarmiento I, Parr TR, Jr, Moeck G, Kreiswirth B. Comparative in vitro activity of oritavancin against recent, genetically diverse, community-associated meticillin-resistant Staphylococcus aureus (MRSA) isolates. Int J Antimicrob Agents. 2010;35(1):934. doi:10.1016/j.ijantimicag.2009.07.012. [PubMed: 19744836]. 
23. US Food and Drug Administration. Oritavancin for the treatment of complicatedskin and skin structure infections. US FDA briefing document for Anti-Infective Drugs Advisory Committee Meeting November 19, 2008; 2008, [5 February 2010]. Available from: http://www.fda.gov/ ohrms/dockets/ac/08/briefing/2008-4394b2-03-FDA.pdf.

24. Belley A, Neesham-Grenon E, McKay G, Arhin FF, Harris R, Beveridge $\mathrm{T}$, et al. Oritavancin kills stationary-phase and biofilm Staphylococcus aureus cells in vitro. Antimicrob Agents Chemother. 2009;53(3):91825. doi: 10.1128/AAC.00766-08. [PubMed: 19104027]. [PubMed Central: PMC2650528].

25. Targanta Therapeutics Corporation. NUVOCID® (oritavancindiphosphateforinjection) for treatment of complicated skin and skin structure infections. NDA 22-153. US FDA Briefing document for the Anti-Infective Drugs Advisory Committee November 19, 2008; 2008, [5 February 2010]. Available from: http://www.fda.gov/ohrms/DOCKETS/ac/ 08/briefing/2008-4394b2-04-Targanta.pdf.

26. Wasilewski MM, Disch DP, McGill JM, Harris HW, O’Riordan WD, Zeckel ML. Equivalence of shorter course therapy with oritavancin vs. vancomycin/cephalexin in complicated skin/skin structure infections. 41st Interscience Conference on Antimicrobial Agents and Chemotherapy (ICAAC).16-19 December 2001; Chicago, IL. Washington, DC. ASMPress; 2001.

27. Giamarellou H, O'Riordan WD, Harris H, Owen S, Porter S, Loutit JS. Phase III trialcomparing 3-7 days of oritavancin vs. 10-14 days of vancomycin/cephalexinin the treatment of patients with complicated skin and skin structure infections(CSSI). 43rd Interscience Conference on Antimicrobial Agents and Chemotherapy (ICAAC). 14-17 September 2003; Chicago, IL. Washington, DC. ASM Press; 2003.

28. Rubino CM, Bhavnani SM, Moeck G, Bellibas SE, Ambrose PG. Population pharmacokinetic analysis for a single 1,200-milligram dose of oritavancin using data from two pivotal phase 3 clinical trials. Antimicrob Agents Chemother. 2015;59(6):3365-72. doi: 10.1128/AAC.00176-15. [PubMed: 25824211]. [PubMed Central: PMC4432154].

29. Corey GR, Kabler H, Mehra P, Gupta S, Overcash JS, Porwal A, et al. Single-dose oritavancin in the treatment of acute bacterial skin infections. N Engl J Med. 2014;370(23):2180-90. doi:10.1056/NEJMoa1310422. [PubMed: 24897083].

30. Boucher HW, Wilcox M, Talbot GH, Puttagunta S, Das AF, Dunne MW. Once-weekly dalbavancin versus daily conventional therapy for skin infection. N Engl J Med. 2014;370(23):2169-79. doi: 10.1056/NEJMoa1310480. [PubMed: 24897082].

31. Seltzer E, Dorr MB, Goldstein BP, Perry M, Dowell JA, Henkel T, et al. Once-weekly dalbavancin versus standard-of-care antimicrobial regimens for treatment of skin and soft-tissue infections. Clin Infect Dis. 2003;37(10):1298-303. doi: 10.1086/379015. [PubMed:14583862].

32. Jauregui LE, Babazadeh S, Seltzer E, Goldberg L, Krievins D, Frederick M, et al. Randomized, double-blind comparison of once-weekly dalbavancin versus twice-daily linezolid therapy for the treatment of complicated skin and skin structure infections. Clin Infect Dis. 2005;41(10):1407-15. doi:10.1086/497271. [PubMed: 16231250].

33. Dunbar LM, Milata J, McClure T, Wasilewski MM; Simplifi Study Team. Comparison of the efficacy and safety of oritavancin front-loaded dosing regimens to daily dosing: An analysis of the SIMPLIFI trial. Antimicrob Agents Chemother. 2011;55(7):3476-84. doi: 10.1128/AAC.00029-11. [PubMed: 21537018]. [PubMed Central: PMC3122413].

34. Deck DH, Jordan JM, Holland TL, Fan W, Wikler MA, Sulham KA, et al. Single-Dose Oritavancin Treatment of Acute Bacterial Skin and Skin Structure Infections: SOLO Trial Efficacy by Eron Severity and Management Setting. Infect Dis Ther. 2016;5(3):353-61. doi: 10.1007/s40121-0160119-9. [PubMed: 27370913]. [PubMed Central: PMC5019975].

35. Corey GR, Arhin FF, Wikler MA, Sahm DF, Kreiswirth BN, Mediavilla JR, et al. Pooled analysis of single-dose oritavancin in the treatment of acute bacterial skin and skin-structure infections caused by Gram-positive pathogens, including a large patient subset with methicillin-resistant Staphylococcus aureus. Int J Antimicrob Agents. 2016;48(5):528-34. doi: 10.1016/j.ijantimicag.2016.07.019. [PubMed:
27665522].

36. US National Library of Medicine. Open-label, dose-finding, pharmacokinetics, safety and tolerability study of oritavancin in pediatric patients with suspected or confirmed bacterial infections. ClinicalTrials.gov. NIH;2010. Available from: https://clinicaltrials.gov/ct2/show/ NCT02134301.

37. Rubinstein E, Lalani T, Corey GR, Kanafani ZA, Nannini EC, Rocha $\mathrm{MG}$, et al. Telavancin versus vancomycin for hospital-acquired pneumonia due to gram-positive pathogens. Clin Infect Dis. 2011;52(1):3140. doi: 10.1093/cid/ciq031. [PubMed: 21148517]. [PubMed Central: PMC3060890].

38. Corey GR, Stryjewski ME, O'Riordan WD, The ATLAS Study Group . ATLAS 1: The first phase 3 study evaluating the new lipoglycopeptide, telavancin, for the treatment of patients with complicated skin and skin structure infections. 17th European Congress of Clinical Microbiology and Infectious Diseases. March 31-April 4, 2007; Munich, Germany. 2007.

39. Corey GR, Stryjewski ME, Fowler VG, Jr, The ATLAS Study Group . ATLAS 2: A double-blind, randomised, active controlled, multinational phase 3 study comparing telavancin with vancomycin for the treatment of patients with complicated skin and skin structure infections. 17th European Congress of Clinical Microbiology and Infectious Diseases. March 31-April 4, 2007; Munich, Germany. 2007.

40. Steele JM, Seabury RW, Hale CM, Mogle BT. Unsuccessful treatment of methicillin-resistant Staphylococcus aureus endocarditis with dalbavancin. J Clin Pharm Ther. 2018;43(1):101-3. doi: 10.1111/jcpt.12580. [PubMed: 28628223].

41. Delaportas DJ, Estrada SJ, Darmelio M. Successful treatment of methicillin susceptible staphylococcus aureus osteomyelitis with oritavancin. Pharmacotherapy. 2017;37(8):e90-2. doi: 10.1002/phar.1957. [PubMed: 28543367].

42. Bhavnani SM, Owen JS, Loutit JS, Porter SB, Ambrose PG. Pharmacokinetics, safety, and tolerability of ascending single intravenous doses of oritavancin administered to healthy human subjects. Diagn Microbiol Infect Dis. 2004;50(2):95-102. doi: 10.1016/j.diagmicrobio.2004.06.007. [PubMed: 15474317].

43. Bhavnani S, Rubino C, Forrest A, Lehoux D, Okusanya OO, Drusano GL. Use of pharmacokinetic-pharmacodynamic (PK-PD) principles to guide clinicaldrug development for oritavancin (ORI). 47th Interscience Conferenceon Antimicrobial Agents and Chemotherapy (ICAAC). 17-20 September 2007; Chicago, IL. Washington, DC. ASM Press; 2007.

44. Moriarty SR, Wasilewski MM, Rosen AS, Perry M. Safety of oritavancinversusvancomycin for treatment of patients with complicated skin and skin-structureinfections. 19th European Congress of Clinical Microbiology and InfectiousDiseases (ECCMID). Helsinki, Finland. Basel, Switzerland. European Society of Clinical Microbiology and Infectious Diseases; 2009.

45. Stryjewski ME, Chu VH, O'Riordan WD, Warren BL, Dunbar LM, Young $\mathrm{DM}$, et al. Telavancin versus standard therapy for treatment of complicated skin and skin structure infections caused by gram-positive bacteria: FAST 2 study. Antimicrob Agents Chemother. 2006;50(3):8627. doi: 10.1128/AAC.50.3.862-867.2006. [PubMed: 16495243]. [PubMed Central: PMC1426424].

46. Leighton A, Gottlieb AB, Dorr MB, Jabes D, Mosconi G, VanSaders $\mathrm{C}$, et al. Tolerability, pharmacokinetics, and serum bactericidal activity of intravenous dalbavancin in healthy volunteers. Antimicrob Agents Chemother. 2004;48(3):940-5. doi: 10.1128/AAC.48.3.940945.2004. [PubMed: 14982787]. [PubMed Central: PMC353075].

47. Dorr MB, Jabes D, Cavaleri M, Dowell J, Mosconi G, Malabarba A, et al. Human pharmacokinetics and rationale for once-weekly dosing of dalbavancin, a semi-synthetic glycopeptide. J Antimicrob Chemother. 2005;55 Suppl 2:ii25-30. doi:10.1093/jac/dki008. [PubMed: 15750034].

48. Marbury T, Dowell JA, Seltzer E, Buckwalter M. Pharmacokinetics of dalbavancin in patients with renal or hepatic impairment.J Clin Pharmacol. 2009;49(4):465-76. doi: 10.1177/0091270008330162. [PubMed: 19318696]. 